\title{
Single-Image Super-Resolution: A Benchmark
}

\author{
Chih-Yuan Yang ${ }^{1}$, Chao $\mathrm{Ma}^{12}$, and Ming-Hsuan Yang ${ }^{1}$ \\ ${ }^{1}$ University of California at Merced \\ ${ }^{2}$ Shanghai Jiao Tong University \\ \{cyang35, cma26, mhyang\}@ucmerced.edu
}

\begin{abstract}
Single-image super-resolution is of great importance for vision applications, and numerous algorithms have been proposed in recent years. Despite the demonstrated success, these results are often generated based on different assumptions using different datasets and metrics. In this paper, we present a systematic benchmark evaluation for state-of-the-art single-image super-resolution algorithms. In addition to quantitative evaluations based on conventional full-reference metrics, human subject studies are carried out to evaluate image quality based on visual perception. The benchmark evaluations demonstrate the performance and limitations of state-of-the-art algorithms which sheds light on future research in single-image super-resolution.
\end{abstract}

Keywords: Single-image super-resolution, performance evaluation, metrics, Gaussian blur kernel width

\section{Introduction}

The goal of single-image super-resolution (SISR) algorithms is to generate highresolution (HR) images from a low-resolution (LR) image input. Numerous SISR algorithms have been recently proposed with different assumptions and evaluation criteria. Broadly speaking, SISR algorithms can be categorized based on their tasks. While domain-specific SISR algorithms focus on specific classes of images such as faces [35, 42], scenes [33], and graphics artwork [18], generic SISR algorithms $[10,38,8,3,27,30,11,46,9,34,32,12,5,39,44,43]$ are developed for all kinds of images where the priors are typically based on primitive image properties such as edges and segments. In order to evaluate the performance of a SISR algorithm, human subject studies or ground truth images are used [33, 43]. In this work, we focus on performance evaluation of state-of-the-art SISR algorithms under different settings based on a set of ground truth images.

Generic SISR algorithms in the literature are usually evaluated with different images and metrics with certain assumptions (e.g., scaling factor and Gaussian kernel width). In addition, the LR images may be generated from different processes (e.g., different downsampling processes). It is thus of great interest to systematically and thoroughly evaluate state-of-the-art SISR algorithms within one framework. For fair comparisons, the ground truth and LR test images should be the same for all evaluated methods. Scaling factors and blur kernel width 
should be considered in performance evaluation. In addition, a wide range of images and metrics should be used for thorough examinations.

In this work, numerous state-of-the-art SISR methods are evaluated systematically and thoroughly. Two large sets of images are used in the experiments. The Berkeley segmentation dataset [20] is widely used for low-level vision problems, and the LIVE1 dataset [28] is commonly used for image quality assessment. We use a wide range of scaling factors and blur kernel width to examine the performance of SISR methods under different assumptions. The HR images generated by SISR methods are evaluated by full-reference metrics and human visual perception. We present the evaluation results and show the limitations of state-of-the-art methods. The findings from these large-scale experiments not only confirm what is commonly believed but also suggest new research directions for SISR. In addition, a code library of state-of-the-art SISR algorithms is available ${ }^{1}$ to the public for ease of reproducing experimental results and evaluating novel algorithms on a common platform.

\section{Related Work}

Generic SISR algorithms aim to generate high-quality HR images from a single LR input image by exploiting certain image priors. According to the image priors, generic SISR algorithms can be categorized into several types of approaches.

Prediction Models. SISR algorithms in this category generate HR images from LR inputs through a predefined mathematical formula without training data. Interpolation-based methods (bilinear, bicubic, and Lanczos) generate HR pixel intensities by weighted averaging neighboring LR pixel values. Since interpolated intensities are locally similar to neighboring pixels, these algorithms generate good smooth regions but insufficient large gradients along edges and at high-frequency regions. The IP method [16] exploits a predefined downsampling model from a HR image to a LR image. Given an initial HR image, this method iteratively generates a LR image through the predefined downsampling model and compensates the difference map in LR back to the HR image. Since a generated HR image is designed to best match the LR input image under the linear downsampling model, the contrast along edges is better enhanced than the results generated by bicubic interpolation.

Edge Based Methods. Edges are important primitive image structures that play a prime role in visual perception. Several SISR algorithms have been proposed to learn priors from edge features for reconstructing HR images. Various edge features have been proposed such as the depth and width of an edge [8] or the parameter of a gradient profile [30]. Since the priors are primarily learned from edges, the reconstructed HR images have high-quality edges with proper sharpness and limited artifacts. However, edge priors are less effective for modeling other high-frequency structures such as textures.

${ }^{1}$ https://eng.ucmerced.edu/people/cyang35 
Image Statistical Methods. Various image properties can be exploited as priors to predict HR images from LR images. The heavy-tailed gradient distribution [15] is exploited in [27] for SISR. The sparsity property of large gradients in generic images is exploited in [17] to reduce the computational load and in [41] to regularize the LR input images. Total variation has also been used as a regularization term for generating HR images $[1,48]$.

Patch Based Methods. Given a set of paired LR and HR training images, patches can be cropped from the training images to learn mapping functions. The exemplar patches can be generated from external datasets [10,2], the input image itself [11,9], or combined sources [44]. Various learning methods of the mapping functions have been proposed such as weighted average [31,2], kernel regression [17], support vector regression [23], Gaussian process regression [13], sparse dictionary representation $[46,7,5,24,45,39,47,19,14]$. In addition to equally averaging overlapped patches, several methods for blending overlapped pixels have been proposed including weighted averaging [11, 44], Markov Random Fields [10], and Conditional Random Fields [38].

\section{Benchmark Settings}

We use two sets of images as the ground truth to thoroughly evaluate the SISR algorithms from diverse sources. From the ground truth HR images, we generate LR test images using various settings of scaling factor and blur kernel width. We generate the SR images by the originally released code $[27,17,46,5,43,36]$ or our implementation $[16,30,11,9]$ if the code is not available. The generated SR image are used to evaluate the performance of SISR algorithms and quality assessment metrics. In order to evaluate the performance of metrics, we conduct human subject studies to generate perceptual scores of the SR images.

Test Image Sets. We use two image sets as the HR ground truth data for evaluation. The first set contains 200 images from the Berkeley segmentation dataset [20], which is widely used for SISR evaluations [11, 9, 32, 12]. All images are of $321 \times 481$ pixels covering diverse contents acquired in a professional photographic style. The second set contains 29 undistorted high-quality images from the LIVE1 dataset [28], which is widely used for image quality assessment [26]. The resolution of these images ranges from $480 \times 720$ to $512 \times 768$ pixels.

Test Image Formation. There are several ways to generate LR test images from the ground truth images $[27,30,36]$ such that the generated LR test images may be numerically different. For clarity, we present an image formulation to address this problem. Given a ground truth HR image $I_{h}$, a scaling factor $s$, and a Gaussian blur kernel width $\sigma$, we generate a test LR image $I_{l}$ by

$$
I_{l}\left(x_{l}, y_{l}\right)=\sum_{x, y} w\left(x-x_{u}, y-y_{u}\right) I_{h}(x, y)+\varepsilon,
$$


Table 1. List of evaluated methods. Language column, M: MATLAB, MC: Mixture of MATLAB and $\mathrm{C} / \mathrm{C}++, \mathrm{E}$ : Executable binary code. Learning column, N: No learning approach involved, E: External exemplar images are required, S: Self-similar exemplars are used. The execution time is measured on a machine with a 2.7 $\mathrm{GHz}$ Quad Core CPU with an image of $128 \times 128$ pixels (shown on the right).

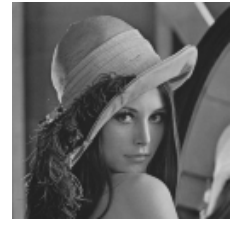

The test image

\begin{tabular}{|l|c|c|c|c|c|c|c|c|}
\hline \multirow{2}{*}{ Method } & \multirow{2}{*}{ Language } & \multirow{2}{*}{ Learning } & \multicolumn{5}{|c|}{ Factors and Execution Time (sec.) } \\
\cline { 5 - 8 } & & & $2 \mathrm{x}$ & $3 \mathrm{x}$ & $4 \mathrm{x}$ & $5 \mathrm{x}$ & $6 \mathrm{x}$ & $8 \mathrm{x}$ \\
\hline \hline Bicubic Interpolation & $\mathrm{MC}$ & $\mathrm{N}$ & 0.002 & 0.002 & 0.003 & 0.004 & 0.004 & 0.005 \\
IP [16] & $\mathrm{M}$ & $\mathrm{N}$ & 0.140 & 0.172 & 0.091 & 0.059 & 0.046 & 0.077 \\
SLJT [27] & $\mathrm{E}$ & $\mathrm{E}$ & 5.913 & 11.90 & 21.29 & 29.19 & 39.78 & 73.49 \\
SSXS [30] & $\mathrm{M}$ & $\mathrm{E}$ & 37.39 & 92.92 & 156.2 & N.A. & N.A. & N.A. \\
GBI [11] & $\mathrm{MC}$ & $\mathrm{S}$ & 364 & 807 & 3851 & 9028 & 21668 & 53762 \\
KK [17] & $\mathrm{MC}$ & $\mathrm{E}$ & 7.715 & 17.14 & 49.06 & N.A. & N.A. & N.A. \\
YWHM [46] & $\mathrm{M}$ & $\mathrm{E}$ & 321 & 598 & 1229 & 1956 & 2477 & 4795 \\
FF [9] & $\mathrm{M}$ & $\mathrm{S}$ & 1779 & 1513 & 2557 & N.A. & N.A. & N.A. \\
DZSW [5] & $\mathrm{M}$ & $\mathrm{E}$ & 266 & 568 & 887 & 1271 & 1721 & 2764 \\
YY [43] & $\mathrm{M}$ & $\mathrm{E}$ & 15.38 & 15.55 & 15.84 & 18.18 & 19.35 & 20.48 \\
TSG [36] & $\mathrm{M}$ & $\mathrm{E}$ & 0.948 & 1.126 & 1.405 & 1.873 & 2.093 & 3.189 \\
\hline
\end{tabular}

where $x_{l} \in\{1, \ldots, m\}$ and $y_{l} \in\{1, \ldots, n\}$ are indices of $I_{l} ; x \in\{1, \ldots, s \times \mathrm{m}\}$ and $y \in\{1, \ldots, s \times \mathrm{n}\}$ are indices of $I_{h}$; and $\varepsilon$ denotes noise. The noise term $\varepsilon$ is introduced from discretization while storing $I_{l}$ into an uncompressed 8-bit image. We compute the HR coordinates $\left(x_{u}, y_{u}\right)$ from the and LR ones $\left(x_{l}, y_{l}\right)$ by

$$
\begin{aligned}
& x_{u}=s\left(x_{l}-0.5\right)+0.5, \\
& y_{u}=s\left(y_{l}-0.5\right)+0.5
\end{aligned}
$$

The weight $w$ is determined by $\sigma$ as

$$
w(\Delta x, \Delta y)=\frac{1}{Z} e^{-\left(\Delta x^{2}+\Delta y^{2}\right) / 2 \sigma^{2}},
$$

where $Z$ is a normalization term. The formation is compatible with most SR methods $[16,11,17,30,46,43,36]$ where the reconstructed images are well aligned with the ground truth images.

Evaluated SISR Methods. For fair comparisons, we evaluate the methods using the original binaries or source code $[27,46,17,5,43,36]$. In addition, we implement four state-of-the-art algorithms when the source or binary code is not available $[16,30,11,9]$. Table 1 lists the evaluated algorithms and their execution time under different scaling factors. We note these methods are implemented in different programming languages. For algorithms where the blur kernel width is an adjustable parameter $[16,27,11,46,5,43]$, we set the same values as used in the LR image formation. We only evaluate the SSXS, KK, and FF methods [30, 17,9 ] under scaling factors 2,3 , and 4 because the released code or priors only 
support these scaling factors. When the training code and dataset are available $[46,5,43,36]$, we re-train the priors for all 54 settings. For algorithms that require other parameter settings $[27,30,46,5,9,43,36]$, the default values in the released code or manuscripts are used.

Human Subject Studies. We conduct human subject studies to evaluate the effectiveness of existing metrics for performance evaluation of SR algorithms. We select 10 images from the BSD200 dataset [20] as the ground truth data. The selected images cover a wide range of high-frequency levels in order to generate a representative subset of the entire BSD200 dataset. (See the supplementary material for their high-frequency levels). From each ground truth image, 9 LR images are generated using Eq. 1 under different settings (the scaling factors of 2,3, and 4, and the Gaussian kernel width of 0.4, 1.2, and 2.0). From each LR image, we use 6 state-of-the-art methods to generate the SR images, and in total we generate $540 \mathrm{SR}$ images. We collect 16,200 perceptual scores from 30 participants evaluating the 540 SR images without knowing the ground truth images or the method names. The SR images are displayed in a random order to avoid bias to favor certain methods. Subjects are asked to give scores between 0 to 10 to assess the image quality based on their visual perception.

\section{Benchmark Evaluation Results}

Fig. 1 shows the quantitative evaluation results using two full-reference metrics PSNR and SSIM, which are widely used in the SISR literature $[2,30,17,46,43$, $36]$. While each row represents one scaling factor, the $x$ and $y$ axes show the Gaussian kernel width $(\sigma)$ and the mean values of all the images in a dataset. We do not show the evaluation results of three test methods (SLJT, DZSW, and $\mathrm{FF}$ ) because the generated SR images do not align with the ground truth images and thus their scores are ineffectively low (the complete comparisons are shown in the supplementary material). The misalignment in the SLJT and DZSW methods is caused by a LR image formation different from Eq. 1 used in our experiments where we sample the central pixels but they sample the pixels at the top-left corner of non-overlapping patches. The FF method uses non-dyadic filter banks to upsample images layer-by-layer in small scaling factors, which does not lead to fully aligned SR images with the ground truth.

Blur Kernel Width and Scaling Factor. Although it is widely accepted that the blur kernel significantly affects the performance of SISR algorithms [21,6], the proper values of blur kernel width have not been thoroughly investigated. Our benchmark evaluations shown in Fig. 1 indicate that the influence of the Gaussian kernel width is consistent across the two test datasets (BSD200 and LIVE1) and the two metrics (PSNR ans SSIM) for various settings of scaling factor and kernel width. Although the settings of peak performance are algorithmdependent, a clear trend can be observed that a larger kernel width is required to generate good performance for a larger scaling factor. According to the experimental results, we suggest to use kernel width with ranges of (0.4-0.6), (0.8-1.0), 


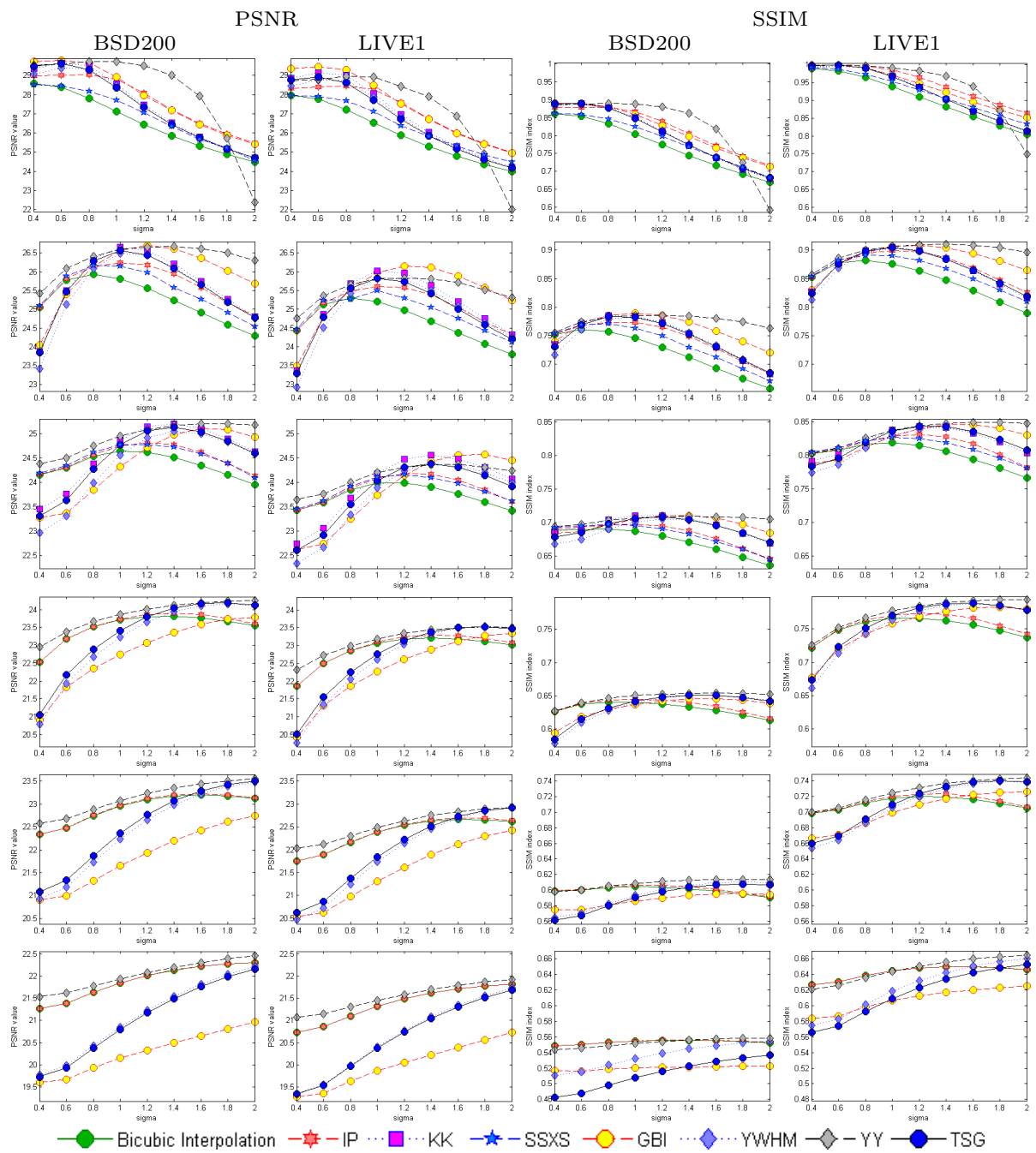

Fig. 1. Performance evaluation. Eight SISR methods are evaluated using two image sets (BSD200 and LIVE1) and two metrics (PSNR and SSIM) under six scaling factors and nine values of Gaussian kernel width. From top to bottom, each row shows results with a scaling factor of $2,3,4,5,6$, and 8 . The plots show mean values for all SR images of a dataset. The BSD200 dataset contains 200 images of $321 \times 481$ pixels, and the LIVE1 dataset contains 29 images ranging from $768 \times 512$ to $480 \times 720$ pixels.

and (1.2-1.4) for scaling factors 2,3 , and 4, respectively, which are different from some given kernel width in the literature [30,43].

We explain these results by considering the LR image formation Eq. 1 where $\sigma$ determines the richness of information preserved in the LR images from the ground truth images. If the value is too large, numerous ground truth pixels will 


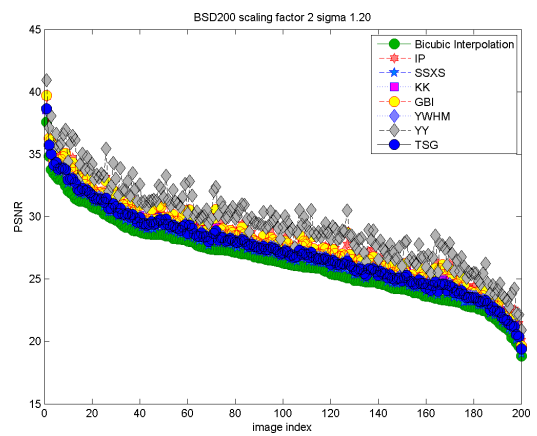

(a) ranked indices

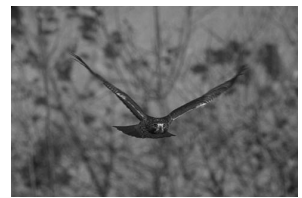

(b) $1 / 37.56$

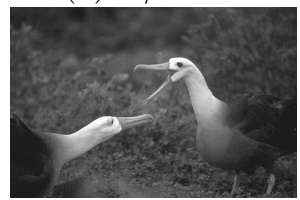

(d) $3 / 33.78$

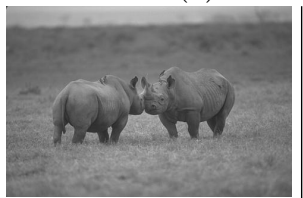

(c) $2 / 34.84$

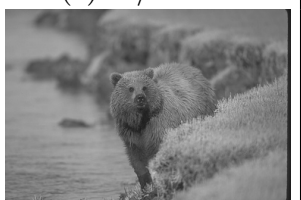

(e) $4 / 33.45$

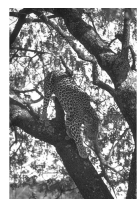

(f) $197 / 20.20$

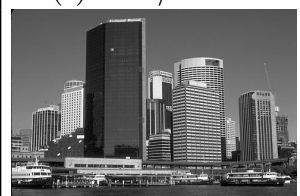

(h) $199 / 19.60$

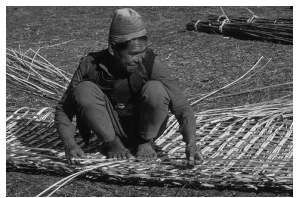

(g) $198 / 19.88$

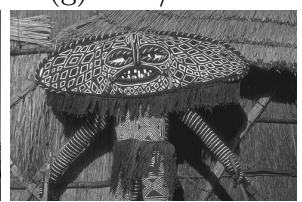

(i) $200 / 18.83$

Fig. 2. Relationship of performance and images. (a) Ranked image indices by the averaged PSNR values of the eight methods for the BSD200 dataset under the setting of the scaling factor of 2 and the Gaussian kernel width of 1.2. (b-i) The ground truth images and their ranked indices / averaged PSNR values. Higher PSNR values can be obtained when images contain fewer high-frequency details. Images best viewed on a high-resolution display with adequate zoom level where each image is shown with at least $320 \times 480$ pixels (full-resolution).

be assigned with similar weights in Eq. 1 and the generated LR image will be blurry, which means that little visual information is preserved for reconstructing an effective HR image similar to the ground truth. If the value is too small, only limited HR pixels will be assigned with large weights and all others are neglected by small weights, which result in reduced information in the LR images. When the $\sigma$ value is properly set for a given scaling factor, most information is preserved in the LR images, which are likely to reconstruct HR images similar to the ground truth images.

Performance Limitations and Potential Solutions. Our human subject studies show that a scaling factor of 4 is the limit of state-of-the-art SISR methods evaluated in this paper. The perceptual scores of upsampled SR images with a scaling factor of 4 are all close to the lower bound designed in the subject studies, which means that the image quality is too low to be evaluated. We in- 
vestigate the reasons of this limitation by exploring the numerical evaluation results which can be viewed as objective quality indices. Fig. 2 shows the ranked results under one setting (the scaling factor of 2 and the Gaussian kernel with of 1.2) of the BSD200 dataset based on the averaged PSNR value of eight SISR methods. An observation is that the performance is primarily determined by the images rather than the SR methods, and we find similar results using the other dataset and metric (SSIM). The image ranks only change slightly even under different settings of scaling factors and Gaussian kernel width. After checking individual images in the dataset, we find that the richness of large magnitude high-frequency details is the key factor. Fig. 2(b)-(e) and Fig. 2(f)-(i) show two sets of images with the highest and lowest PSNR indices where images with more highly contrast pixels lead to smaller PSNR values, and vice versa.

We explain the performance limitation of the evaluated SR algorithms. Except the bicubic interpolation and IP [16] methods, all SR algorithms rely on statistical priors to predict HR features from LR ones. The priors of cross-scale self-similarity used in the GBI method [11] is also a specialized statistical prior as shown in $[9,49]$. In order to process a wide range of images and to train the priors with limited computational resources, the features of the tested algorithm are all extracted from small patches. According to the statistics of natural images [15], the patches containing large magnitude of gradients are rare in natural images. Since a LR patch can be generated from various HR patches through the downsampling process of Eq. 1, any learned statistical priors must be able to generate predicted HR features close to the majority of the training HR features for high-quality SR images. As a result, rare patches are less likely to be effectively reconstructed and this leads to low PSNR and SSIM indices.

These findings are useful for developing effective SR algorithms. First, it is useful to divide training data into non-overlapping subsets where HR features of large magnitude of gradients will not be averaged by other features significantly. Such an approach has been adopted in $[43,36]$ and our experimental results show that the algorithm performs well against other algorithms in most settings. Second, it is reasonable to enlarge the feature dimension in order to increase the distinguishability of LR patches. This approach is used in [32] where patches are extended to segments in order to reconstruct effective high-frequency details. However, the ensuing high computational load and large amount of training data will be challenging for this approach to scale up due to the curse of dimensionality. Third, it is ideal to classify training data and to analyze image contents to facilitate specific priors for each class. This idea has been used in $[12,42]$ for specific domains such as textures and faces. However, it remains an open question how to generate a sufficient number of classes and parse images for generic SR.

Evaluation Metrics. The PSNR and SSIM index are the most widely used metrics in SR problems, but they do not reflect image quality well [44]. As explained in Section 3, we conduct human subject studies to validate the effectiveness of metrics for SR images. Fig. 3 shows the relationship between perceptual scores on the $\mathrm{X}$ axes and metric indices on the $\mathrm{Y}$ axes. Effective metrics should generate 


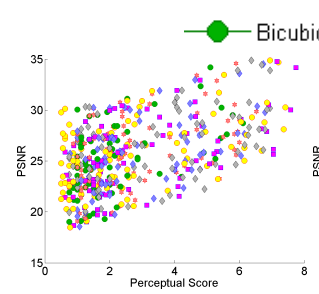

(a) PSNR, $\rho=0.4760$

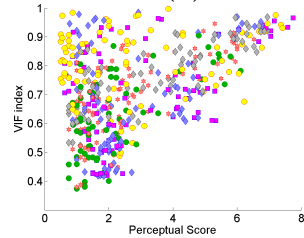

(c) VIF, $\rho=0.3874$

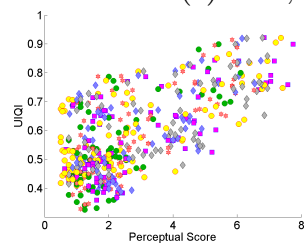

(e) UIQI, $\rho=0.6060$

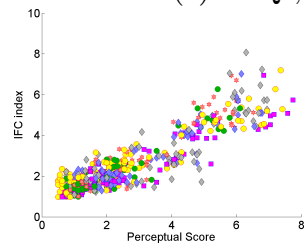

(g) IFC, $\rho=0.8434$
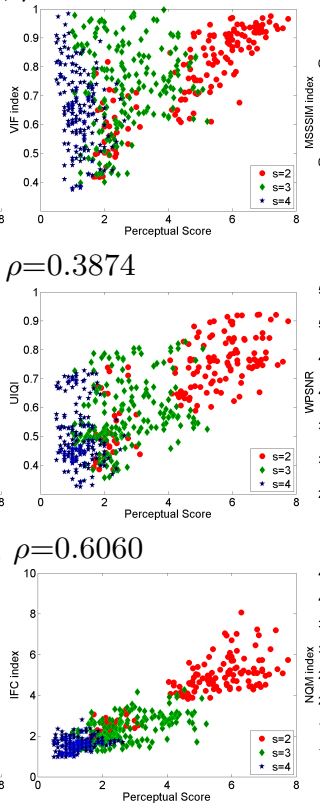

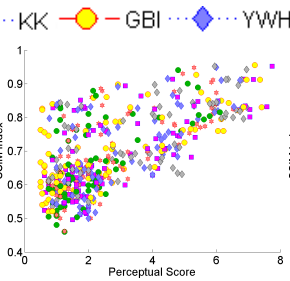

(b) SSIM, $\rho=0.6203$
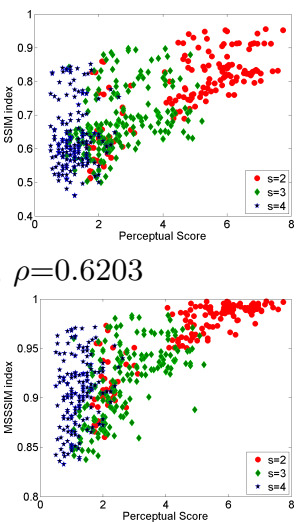

(d) MSSSIM, $\rho=0.7094$
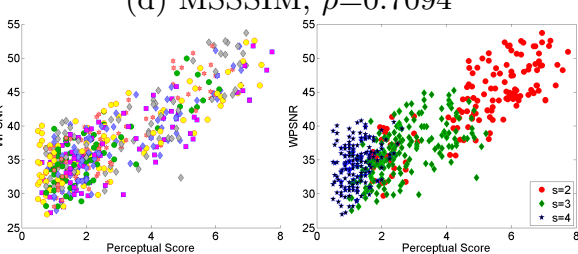

(f) WPSNR, $\rho=0.7446$
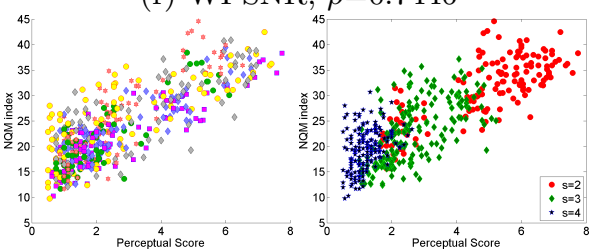

(h) NQM, $\rho=0.7632$

Fig. 3. Relationship between perceptual scores and metric indices for eight image quality metrics. Since the metric ranges are different, we compute the performance by the Spearman's rank correlation coefficient (denoted by $\rho$ ), which is not affected by the ranges. The left and right plots distinguish from different SR methods and scaling factors (denoted by $s$ ) by colors, which are best viewed on a color display. Experimental results show that SR images should be evaluated by the IFC metric due to the better effectiveness instead of the widely used metrics PSNR and SSIM.

quality indices where the ranks are similar to the ones of perceptual scores. We evaluate the performance of a metric by the Spearman's rank correlation coefficient [22], which is not affected by different ranges of quality indices generated by different metrics.

Our experimental results indicate that for SR images four metrics are more effective such as the multi-scale structure similarity index (MSSSIM) [40], information fidelity criterion (IFC) [29], weighted peak signal-to-noise ratio (WPSNR), and noise quality measure (NQM) [4]. Overall, the IFC index has highest cor- 
relation with perceptual scores for SR evaluation. We examine the effectiveness of the IFC metric for SR images by the extracted features and specialized application for natural images. First, the IFC metric is designed to evaluate the loss of image information so that it extracts wavelet features with focus on highfrequency details rather than low-frequency components. This metric matches human perception well as visual perception is more sensitive to high-frequency details of SR images rather than low-frequency components. Second, the IFC metric is developed based on natural scene statistics using the Gaussian scale mixtures [37] and the BSD200 dataset contains numerous such images.

We note that the extracted features by the VIF method put more weight on edges which are of great importance for visual perception. As a result, SR images with sharp edges have large VIF values even though they are not visually pleasant. Fig. 3(c) shows that many points in the upper left region have low perceptual scores but high VIF indices, especially for images generated by the GBI method. We compare a set of the generated images in Fig. 4 where the one generated by the GBI method contains apparently over-sharpened edges than other methods, which indicates the VIF metric is not effective for SR performance evaluation. It is worth noticing that the weights computed in the WPSNR metric significantly improve the performance over the widely used PSNR metric (where the weights can be viewed as the same). The weights of WPSNR in our experiments are computed by a function [25] that models contrast sensitivity of perception in terms of spatial frequency. As a result, patches in a SR image carrying signals $\mathrm{n}$ mid frequency ranges will be assigned with larger weights, and the smooth regions and complicated textures will have smaller weights. Thus the WPSNR metric performs well with the assigned weights.

Evaluations of SISR Methods. As shown in Table 1, the bicubic interpolation and IP [16] methods perform well with low computational load. The IP method almost always outperforms the bicubic interpolation method in terms of visual quality by iteratively restoring high-frequency details based on a difference map between the LR test image and a downsampled image of the estimated HR result. The restored high-frequency map enhances contrast of edges and textures, and makes a SR image more similar to the ground truth image than the one generated by bicubic interpolation. However, the IP method is limited by the accuracy of the restored high-frequency map. Since it is simply interpolated from a LR difference map, the HR results are better when the compensated difference is limited with small scaling factor as shown in Fig. 1. The low computational load of the back-projection method makes it widely used as a post-processing step to refine contrast in state-of-the-art SISR methods $[11,46]$.

The GBI method [11] generates sharp contours as it uses a small scaling factor (1.25) to upsample intermediate images in a pyramid. The exploited selfexemplar priors are effective for contour patches because the image structures remain similar after being downsampled with a small scaling factor. Furthermore, it utilizes the back-projection compensation to enhance the contrast in every upsampling iteration. Due to the generated sharp contours, the GBI method works well for some settings such as scaling factor of 3 with $\sigma$ greater than 0.6 , and the 


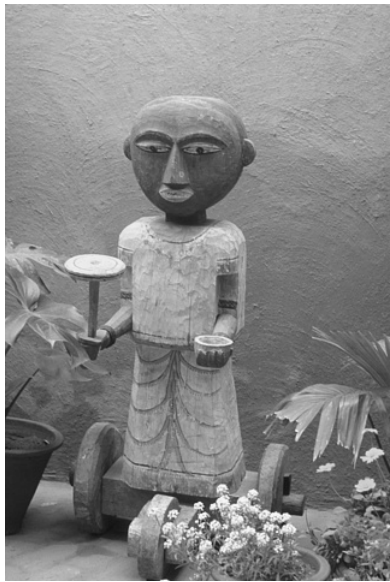

(a) Ground truth

Perceptual score/VIF index

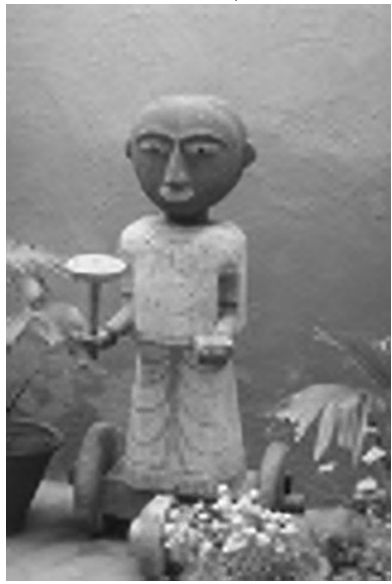

(d) YY [43]

$2.00 / 0.7208$

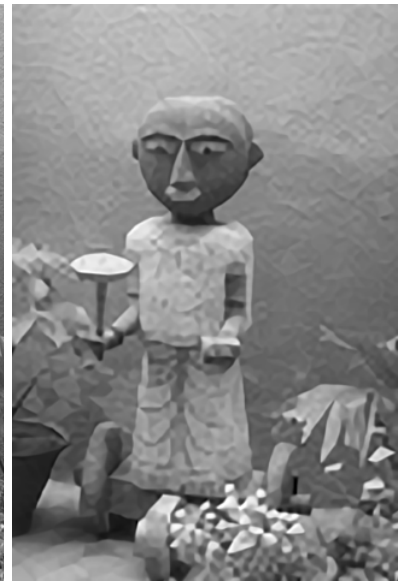

(b) GBI [11]

$1.88 / 0.8891$

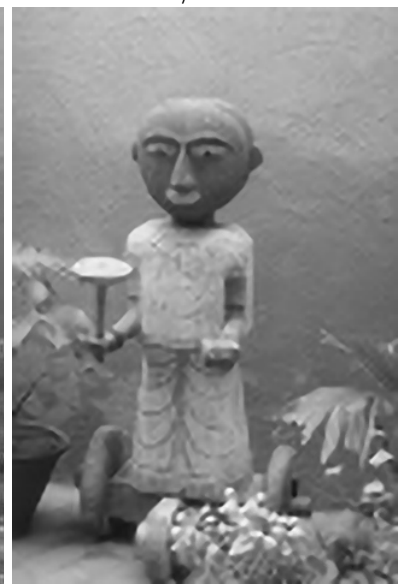

(e) $\mathrm{KK}[17]$

$2.5 / 0.6880$

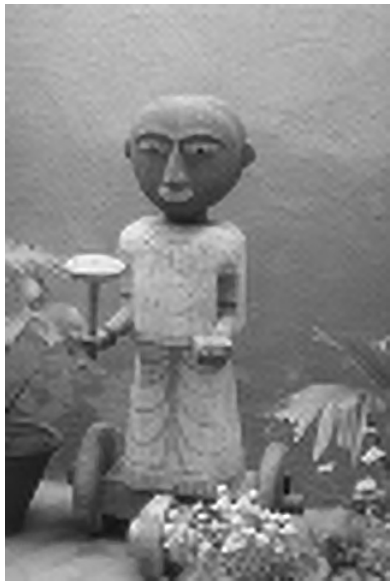

(c) YWHM [46]

$1.63 / 0.7226$

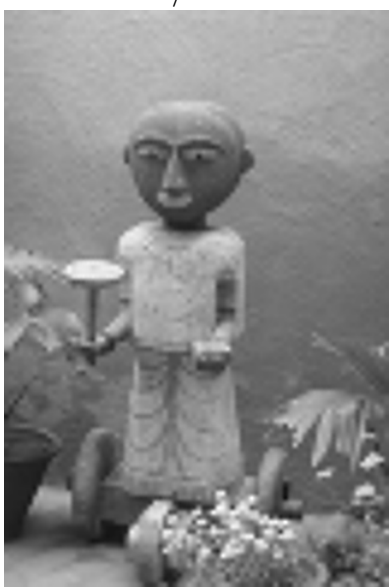

(f) IP [16]

$2.00 / 0.6554$

Fig. 4. A set of super-resolution images and their perceptual scores and VIF indices. (b)-(f) Five SR images generated under the same setting (the scaling factor of 4, and the Gaussian kernel width of 1.2). Since the VIF metric uses edge features to evaluate image quality, images with sharp edges likely generate large VIF indices. The GBI method generates over-sharpened edges in (b) compared to the ground truth image in (a) and distorted image structures like the flowers at the bottom, which lead to a low perceptual score but a large VIF index. Images best viewed on a high-resolution display with adequate zoom level where each image is shown with at least $320 \times 480$ pixels (full-resolution).

scaling factor of 4 with $\sigma$ greater than 1.4. However, the performance of the GBI method is limited because of three factors. First, this method exploits self-similar exemplar patches only from the input LR image through a pyramid. When the 
scaling factor is large, it may be difficult for a patch to find similar ones in the exemplar set through the pyramid (i.e., lack of sufficient exemplar patches). Second, the repeated usage of the back-projection results in over-contrasted contours. As shown in Fig. 4(b), the contours along the face and arms of the wood figure are over-contrasted, and the image structures of the flowers at the bottom are distorted. Thus the performance of the GBI method decreases significantly when the scaling factor is greater than 4 (more details can be found in the supplementary material). Third, the method requires high computational load as a result of searching for similar patches in a pyramid. As shown in Table 1, it is the most computationally expensive method among all.

The YWHM, YY, and TSG methods $[46,43,36]$ all upsample high-frequency components (pixel gradients or patch difference) from LR to HR through learned mapping functions, and the main difference is about learning approaches. While the YWHM and TSG methods learn a pair of sparse dictionaries, the YY method trains numerous simple linear functions. The difference between the YWHM and TSG methods is usage of the dictionaries. Indeed the YWHM method uses the dictionaries to generate sparse coefficients while the TSG method uses the dictionaries as sets of anchor points in LR/HR feature spaces, which is more similar to the YY method in this manner. Since the computational load of generating sparse coefficients is skipped, the TSG method gains significant advantages on execution time over the YWHM method as shown in Table 1 while their performance is similar as shown in Fig. 1.

We discuss the difference of the YY and TSG methods since both partition the LR feature space into numerous subspaces in order to map LR features to HR space by individual linear functions. The most significant difference lies in anchor points used to partition the LR feature space where they are evenly scattered in the YY method due to the L2-norm distance but restricted in a unit sphere in the TSG method because they are all bases of a sparse dictionary. The difference of anchor points lead to the differences of computational load and performance. The unit-length anchor points used in the TSG method have advantages on computation in which the cost of finding anchor points is lower as highest correlation can be easily computed by inner product. However, this step in the YY method is computed by L2-norm distances which is computationally more expensive. On the contrary, the evenly scattered anchor points used in the YY method lead to better performance for most settings as shown in Fig. 1 because the regression functions can be learned more directly and effectively. As features extracted from training images are directly grouped by the evenly scattered anchor points, the source patches are visually similar which in turn facilitates learning better regression functions.

We find that the YY method performs poorly when the scaling factor is 2 and the $\sigma$ value is 2.0, which can be attributed to sensitivity of the learned functions. When the scaling factor is small but $\sigma$ is large, most generated LR patches are smoothed, and thus the feature values of difference patches are close to zero. However, the HR source patches are highly varied and the feature values are large. As a result, this linear regression model is likely to have a large condition 


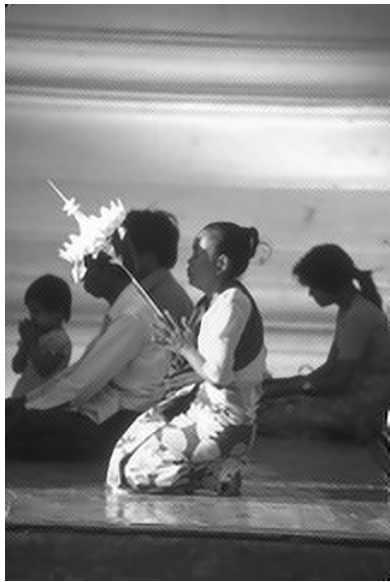

(a) $\sigma=1.6$

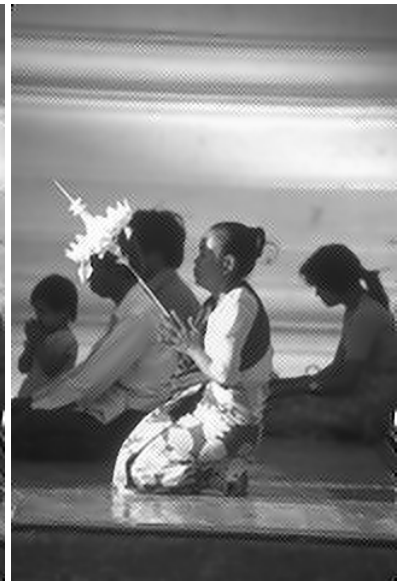

(b) $\sigma=1.8$

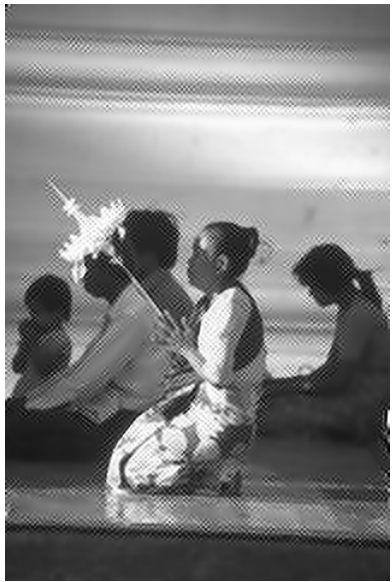

(c) $\sigma=2.0$

Fig. 5. A set of super-resolution images to show the sensitivity of learned regression functions in the YY method [43]. (a)-(c) The ground truth image and scaling factor of 2 are the same while the only difference is the parameter of Gaussian kernel width $(\sigma)$ used in the low-resolution image formation Eq. 1. The artifacts caused by sensitivity of linear regression can be found along contours. Images best viewed on a high-resolution display with adequate zoom level where each image is shown with at least $320 \times 480$ pixels (full-resolution).

number and leads to numerically instability. As shown in Fig. 5, the artifacts are the noise caused from many high-frequency details.

\section{Conclusion}

In this paper, several state-of-the-art SISR methods are thoroughly studied. Hundreds of images are evaluated using various scaling factors and Gaussian kernel width values. Comprehensive experimental results show how state-of-theart SISR methods perform with respect to scaling factor, Gaussian kernel and image contents. The benchmark evaluations demonstrate the performance and limitations of state-of-the-art algorithms quantitatively and qualitatively. The developed code library of state-of-the-art SISR algorithms provides a common platform for ease of reproducing experimental results and evaluating novel algorithms for future research.

\section{Acknowledgment}

This work is supported in part by the NSF CAREER Grant \#1149783 and NSF IIS Grant \#1152576. C. Ma is sponsored by CSC fellowship. 


\section{References}

1. Aly, H.A., Dubois, E.: Image up-sampling using total-variation regularization with a new observation model. TIP 14(10), 1647 -1659 (2005)

2. Chang, H., Yeung, D.Y., Xiong, Y.: Super-resolution through neighbor embedding. In: CVPR (2004)

3. Dai, S., Han, M., Xu, W., Wu, Y., Gong, Y.: Soft edge smoothness prior for alpha channel super resolution. In: CVPR (2007)

4. Damera-Venkata, N., Kite, T.D., Geisler, W.S., Evans, B.L., Bovik, A.C.: Image quality assessment based on a degradation model. TIP 9(4), 636-650 (2000)

5. Dong, W., Zhang, L., Shi, G., Wu, X.: Image deblurring and super-resolution by adaptive sparse domain selection and adaptive regularization. TIP 20(7), 1838 1857 (2011)

6. Efrat, N., Glasner, D., Apartsin, A., Nadler, B., Levin, A.: Accurate blur models vs. image priors in single image super-resolution. In: ICCV (2013)

7. Elad, M., Zeyde, R., Protter, M.: Single image super-resolution using sparse representation. In: SIAM Imaging Science (2010)

8. Fattal, R.: Image upsampling via imposed edge statistics. In: SIGGRAPH (2007)

9. Freedman, G., Fattal, R.: Image and video upscaling from local self-examples. TOG 30(2), 1-11 (2011)

10. Freeman, W.T., Jones, T.R., Pasztor, E.C.: Example-based super-resolution. IEEE Computer Graphics and Applications pp. 56-65 (March/April 2002)

11. Glasner, D., Bagon, S., Irani, M.: Super-resolution from a single image. In: ICCV (2009)

12. HaCohen, Y., Fattal, R., Lischinski, D.: Image upsampling via texture hallucination. In: ICCP (2010)

13. He, H., Siu, W.C.: Single image super-resolution using Gaussian process regression. In: CVPR (2011)

14. He, L., Qi, H., Zaretzki, R.: Beta process joint dictionary learning for coupled feature spaces with application to single image super-resolution. In: CVPR (2013)

15. Huang, J., Mumford, D.: Statistics of natural images and models. In: CVPR (1999)

16. Irani, M., Peleg, S.: Improving resolution by image registration. CGVIP 53(3), 231-239 (1991)

17. Kim, K.I., Kwon, Y.: Single-image super-resolution using sparse regression and natural image prior. PAMI 32(6), 1127 -1133 (2010)

18. Kopf, J., Lischinski, D.: Depixelizing pixel art. In: SIGGRAPH (2011)

19. Lu, X., Yuan, H., Yan, P., Yuan, Y., Li, X.: Geometry constrained sparse coding for single image super-resolution. In: CVPR (2012)

20. Martin, D., Fowlkes, C., Tal, D., Malik, J.: A database of human segmented natural images and its application to evaluating segmentation algorithms and measuring ecological statistics. In: ICCV (2001)

21. Michaeli, T., Irani, M.: Nonparametric blind super-resolution. In: ICCV (2013)

22. Moore, D., McCabe, G.P., Craig, B.: Introduction to the Practice of Statistics. W. H. Freeman, 7th edn. (2005)

23. Ni, K., Nguyen, T.: Image superresolution using support vector regression. TIP 16(6), 1596-1610 (2007)

24. Purkait, P., Chanda, B.: Image upscaling using multiple dictionaries of natural image patches. In: ACCV (2012)

25. ROBSON, J.G.: Spatial and temporal contrast-sensitivity functions of the visual system. Journal of the Optical Society of America (1966) 
26. Saad, M.A., Bovik, A.C., Charrier, C.: Blind image quality assessment: A natural scene statistics approach in the DCT domain. TIP 21(8), 3339-3352 (2012)

27. Shan, Q., Li, Z., Jia, J., Tang, C.K.: Fast image/video upsampling. In: SIGGRAPH Asia (2008)

28. Sheikh, H.R., Sabir, M.F., Bovik, A.C.: A statistical evaluation of recent full reference image quality assessment algorithms. TIP 15(11), 3340-3451 (2006)

29. Sheikh, H.R., Bovik, A.C., de Veciana, G.: An information fidelity criterion for image quality assessment using natural scene statistics. TIP 14(12), 2117-2128 (2005)

30. Sun, J., Sun, J., Xu, Z., Shum, H.Y.: Image super-resolution using gradient profile prior. In: CVPR (2008)

31. Sun, J., Zheng, N.N., Tao, H., Shum, H.Y.: Image hallucination with primal sketch priors. In: CVPR (2003)

32. Sun, J., Zhu, J., Tappen, M.F.: Context-constrained hallucination for image superresolution. In: CVPR (2010)

33. Sun, L., Hays, J.: Super-resolution from internet-scale scene matching. In: ICCP (2012)

34. Tai, Y.W., Liu, S., Brown, M.S., Lin, S.: Super resolution using edge prior and single image detail synthesis. In: CVPR (2010)

35. Tappen, M.F., Liu, C.: A Bayesian approach to alignment-based image hallucination. In: ECCV (2012)

36. Timofte, R., Smet, V.D., Gool, L.V.: Anchored neighborhood regression for fast example-based super-resolution. In: ICCV (2012)

37. Wainwright, M.J., Simoncelli, E.P., Willsky, A.S.: Random cascades on wavelet trees and their use in analyzing and modeling natural images. Applied and Computational Harmonic Analysis 11, 89-123 (2001)

38. Wang, Q., Tang, X., Shum, H.: Patch based blind image super resolution. In: ICCV (2005)

39. Wang, S., Zhang, L., Liang, Y., Pan, Q.: Semi-coupled dictionary learning with applications to image super-resolution and photo-sketch synthesis. In: CVPR (2012)

40. Wang, Z., Simoncelli, E., Bovik, A.C.: Multi-scale structural similarity for image quality assessment. In: IEEE Conference Record of the Thirty-Seventh Asilomar Conference on Signals, Systems, and Computers (2003)

41. Xiong, X., Sun, X., Wu, F.: Robust web image/video super-resolution. TIP 19(8), 2017-2028 (2010)

42. Yang, C.Y., Liu, S., Yang, M.H.: Structured face hallucination. In: CVPR (2013)

43. Yang, C.Y., Yang, M.H.: Fast direct super-resolution by simple functions. In: ICCV (2013)

44. Yang, J., Lin, Z., Cohen, S.: Fast image super-resolution based on in-place example regression. In: CVPR (2013)

45. Yang, J., Wang, Z., Lin, Z., Shu, X., Huang, T.: Bilevel sparse coding for coupled feature spaces. In: CVPR (2012)

46. Yang, J., Wright, J., Huang, T., Ma, Y.: Image super-resolution via sparse representation. TIP (2010)

47. Yang, S., Wang, M., Chen, Y., Sun, Y.: Single-image super-resolution reconstruction via learned geometric dictionaries and clustered sparse coding. TIP 21(9), 4016-4028 (2012)

48. Zhang, H., Yang, J., Zhang, Y., Huang, T.S.: Non-local kernel regression for image and video restoration. In: ECCV (2010)

49. Zontak, M., Irani, M.: Internal statistics of a single natural image. In: CVPR (2011) 